\title{
PLANAR SHAPE MANIPULATION USING APPROXIMATE GEOMETRIC PRIMITIVES
}

\author{
Victor Milenkovic*and Elisha Sacks ${ }^{\dagger}$ and Steven Trac ${ }^{\ddagger}$
}

November 8, 2018

\begin{abstract}
We present robust algorithms for set operations and Euclidean transformations of curved shapes in the plane using approximate geometric primitives. We use a refinement algorithm to ensure consistency. Its computational complexity is $\mathrm{O}(n \log n+k)$ for an input of size $n$ with $k=\mathrm{O}\left(n^{2}\right)$ consistency violations. The output is as accurate as the geometric primitives. We validate our algorithms in floating point using sequences of six set operations and Euclidean transforms on shapes bounded by curves of algebraic degree 1 to 6 . We test generic and degenerate inputs.
\end{abstract}

\section{Introduction}

Set operations and Euclidean transformations are important computational geometry tasks with many applications. There are efficient algorithms for planar shapes, which are more common and simpler than 3D shapes. A shape is modeled as a subdivision: a partition of the plane into faces, curves, and points. Fig. 1. 1 shows a subdivision with faces $f_{0}$ and $f_{1}$, curves $e_{1}, \ldots, e_{4}$, and points indicated with dots. A set operation is performed by constructing the mutual refinement of the input subdivisions, called the overlay, and returning the faces that satisfy the set operator (Fig. 15). A Euclidean transformation is performed by transforming the points, curves, and faces of the input subdivision.

Shape manipulation algorithms use geometric primitives that are formulated in the real-RAM model where real arithmetic is exact and has unit cost. The robustness problem is how to implement these primitives in computer arithmetic. The mainstream strategy is to implement primitives exactly using integer arithmetic and algebraic computation. We prefer approximate primitives in floating

\footnotetext{
* Department of Computer Science, University of Miami. Coral Gables, FL 33124-4245, USA.vjm@cs.miami.edu

${ }^{\dagger}$ Computer Science Department, Purdue University. West Lafayette, IN 47907-2066, USA. eps@cs.purdue.edu

$\ddagger$ Department of Computer Science, University of Miami. Coral Gables, FL 33124-4245, USA.strac@cs.miami.edu
} 


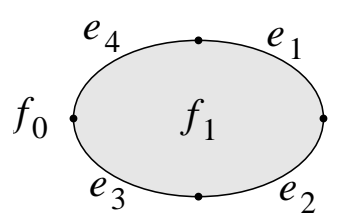

(a)

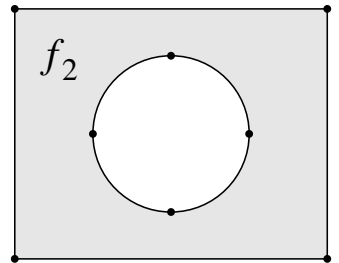

(b)

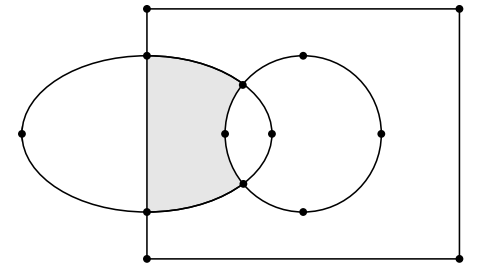

(c)

Figure 1: Subdivisions (a, b) and their overlay (c) with $f_{1} \cap f_{2}$ shaded.

point because they are much faster and have constant bit complexity. Although the approximation error is negligible, it can cause prior shape manipulation algorithms to generate inconsistent or highly inaccurate outputs. We present shape manipulation algorithms that use approximate primitives yet generate consistent, accurate outputs.

\section{$1.1 \quad$ Prior work}

Yap[1] describes exact computational geometry. Exact computation increases bit complexity, hence running time. The CGAL library 2] provides floating point filtering techniques that somewhat reduce this cost. The increase in bit complexity from input to output leads to unbounded complexity in sequences of computations. The only current solution is to simplify the output. Although simplification algorithms have been developed for a few domains, [3, 4, 5, 6] not including implicit algebraic curves, a general strategy is unknown.

The alternative to exact primitives is approximate primitives. The robustness problem is to ensure consistency despite approximation error.

The controlled perturbation robustness strategy is to verify that the predicates in an algorithm are correct despite approximation error. Verification fails when the predicate value is too close to zero. Failure is prevented probabilistically by randomly perturbing the input parameters. If any predicate still cannot be verified, the input is perturbed again. Controlled perturbation has been applied to arrangements, [9, 10, 11] convex hulls, 12] and Delaunay triangulation. 12 The perturbation size is exponential in the algebraic degree

of the primitives. The degree-four perturbation size exceeds the error bounds of engineering applications. We developed versions of controlled perturbation that solve this problem and used them to compute Minkowski sums of polyhedra[13] and free spaces of planar robots. 14] Controlled perturbation does not work for shape manipulation because it does not handle algebraic curves and does not address output simplification in sequences of computations.

Our robustness strategy is to eliminate inconsistency due to approximate geometric primitives by enforcing consistency constraints on the data structures that represent geometric objects. We analyze the running time and the accuracy in terms of the approximation error, $\delta$, and the number of constraint violations, $k$, but neither parameter is provided to the algorithms. An algorithm is incon- 
sistency sensitive when the extra running time for inconsistency elimination is polynomial in $k$ and $n$, the extra error is polynomial in $k$ and $n$ and is linear in $\delta$, and $k$ is polynomial in $n$. Inconsistency sensitivity captures the concept of an efficient approximate algorithm: fast and accurate when there are few inconsistencies, and degrading gracefully.

We developed an inconsistency sensitive arrangement algorithm for algebraiccurve segments. 7] The $x$-coordinates where segments cross and the segment $y$ order between crossings are computed with $(1+k n) \epsilon$ accuracy for $n$ segments, where $\epsilon$ denotes the error in the approximate geometric primitives of that algorithm. A consistency constraint violation is a cyclic $y$-order, due to an incorrect ordering of segment crossing coordinates. The algorithm computes a consistent $y$-order of size $V=2 n+N+\min \left(3 k n, n^{2} / 2\right)$ for $N$ crossings. The running time is $\mathrm{O}(V \log n)$ and the output is correct for a set of segments within $(1+k n) \epsilon$ of the output segments. We also developed two inconsistency sensitive Minkowski sum algorithms. 8

The arrangement algorithm can compute a curve $y$-order that is inconsistent with the curve endpoints, as illustrated below. In prior work, we developed a simple solution for the special case where every curve endpoint has a distinct $x$-coordinate. We handled the general case heuristically by sweeping along a random axis. This strategy proved inadequate even for generic inputs. In this paper, we present a general, inconsistency sensitive solution.

\subsection{Inconsistency in the Overlay Algorithm}

Our overlay algorithm uses three geometric primitives: computing intersection points of monotone curves, computing turning points of non-monotone curves, and comparing coordinates of points. The first primitive is required by any overlay algorithm, while the other two are dictated by our use of a sweep algorithm. The high level algorithm is as follows. Split the curves at their turning points. Split the monotone curves at their intersection points to obtain sub-curves. Derive the $y$-order of each pair of sub-curves that overlap in $x$ by comparing the $y$ coordinates of their intersection points with a vertical line at the middle of their interval of $x$-overlap. The details of the sweep and how it avoids creating cycles appears in previous work 8 and in Sec. 6.

The algorithm output, the $x$-order of the curve endpoints and the partial $y$ order of the sub-curves, allows us to compute the subdivision structure without additional approximate primitives. In particular, we can use a standard sweep algorithm to generate a vertical cell decomposition. The curve endpoints are the sweep events in order of increasing $x$. The sweep list contains curves in $y$-order whose left endpoint event has been processed and whose right endpoint has not. If a subdivision construction algorithm, e.g. randomized incremental vertical cell decomposition, requires endpoint/curve order, the $y$-order of endpoint $a$ of curve $a b$ with respect to curve $c d$, with $c_{x}<a_{x}<d_{x}$, is deduced from the calculated $y$-order of $a b$ and $c d$.

We illustrate that an inconsistent $y$-order can arise in computing the overlay of a monotone curve, $e_{2}$, with two monotone curves, $e_{1}$ and $e_{3}$, that meet at a 


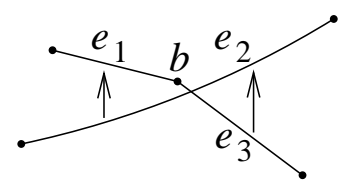

(a)

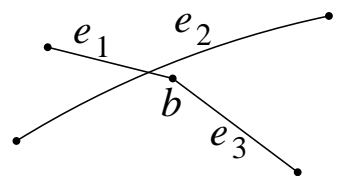

(b)

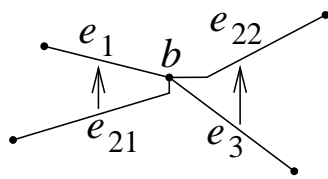

(c)

Figure 2: Inconsistent $y$-order (a), $e_{2}$ deformation (b), and consistent refinement (c).

point, $b$ (Fig. 2a). Due to numerical error, the intersection primitive incorrectly reports that $e_{2}$ and $e_{3}$ do not intersect. Intersections with vertical lines (arrows) order $e_{1}$ above $e_{2}$ and $e_{2}$ above $e_{3}$. The incorrect $y$-order of $e_{2}$ and $e_{3}$ is correct for a small deformation of the input (Fig. 2 b). But the algorithm output is inconsistent because no deformation can make $e_{2}$ be below $e_{1}$, above $e_{3}$, and disjoint from $b$.

\subsection{Contribution and Organization}

Our main result is an inconsistency sensitive refinement algorithm that assigns a consistent $y$-order to a set of curves by splitting some curves at endpoints of other curves (Fig. 2r). The output $y$-order is the induced refinement of the input $y$-order. In our example, $e_{2}$ is split into $e_{21}$ and $e_{22}$ at $b, e_{21}$ is below $e_{1}$, $e_{22}$ is above $e_{3}$, and the other pairs are unordered because their domains are disjoint. The precondition is that each pair of input curves can be placed in the input $y$-order using a deformation of at most $\delta$. The postcondition is that a single $\delta$-deformation can place all the output curves in the output $y$-order. The running time is $\mathrm{O}(n \log n+k)$ for $n$ curves with $k=\mathrm{O}\left(n^{2}\right)$ consistency violations. We define concepts in Sec. 2 present the refinement algorithm in Sec. 3, and analyze it in Secs. 4 囵

As indicated above, overlay can introduce inconsistencies. Surprisingly, even transformations can do so. To accomplish set operations, we start with a possibly inconsistent overlay, run the refinement algorithm, construct the faces, and extract the relevant faces (Sec. 6). To transform a subdivision, we transform its curves, split rotated curves at new turning points, run refinement, and construct the faces (Sec. 7). In this manner, the refinement algorithm enables us to accomplish consistent and accurate shape manipulation using approximate geometric primitives.

We present an empirical validation that our algorithms are fast and accurate on sequences of six set operations and Euclidean transformations on shapes bounded by curves of algebraic degree 1 to 6 (Sec. 8). Moreover, $k$ is zero for generic input and is small for degenerate input. We conclude with a discussion (Sec. 9). 


\section{Concepts}

In this section, we define approximate subdivisions and four ways they can be inconsistent. Fig. 2 $2 \mathrm{a}$ is an example of an approximate subdivision and the depicted inconsistency is an instance of one of these ways. The refinement algorithm eliminates these inconsistencies. We prove that the output represents a consistent shape (Sec. 4) and that its error is bounded by the input error (Sec. 5). Each group of related concepts is defined informally then formally.

We work in the $x y$ plane. A point, $a$, has Cartesian coordinates $\left(a_{x}, a_{y}\right)$. Curves are open, bounded, and piecewise algebraic. They are assumed $x$ monotone and $y$-monotone unless stated otherwise. A curve, $e$, whose endpoints are $a$ and $b$ with $a_{x} \leq b_{x}$ is denoted $e[a, b]$. It is vertical when $a_{x}=b_{x}$, horizontal when $a_{y}=b_{y}$, increasing when $a_{y}<b_{y}$, and decreasing when $a_{y}>b_{y}$.

We represent a subdivision as a set of disjoint curves (Def. 1). The combinatorial structure of the subdivision is derivable from the $x$-order of the curve endpoints and the $<_{y}$ partial order on the curves (Def. 3), as explained in the introduction.

Definition 1 (subdivision). A subdivision is a set of curves in which every member is disjoint from the closure of every other member.

Definition 2. Sets $s$ and $t$ overlap in $x$ if there exist $p \in s$ and $q \in t$ with $p_{x}=q_{x}$.

Definition $3\left(<_{y}\right)$. For two point sets, $s$ and $t$, that overlap in $x, s<_{y} t$ if $p_{y}<q_{y}$ for each $p \in s$ and $q \in t$ with $p_{x}=q_{x}$. For a point, $a, a<_{y} s$ means $\{a\}<{ }_{y} s$.

We model a shape with an approximate subdivision: a set of curves with an imposed acyclic partial $y$-order (Def. (4) denoted $\prec_{y}$. An approximate subdivision is a subdivision when its curves are disjoint from the closures of other curves and the $\prec_{y}$ order matches the $<_{y}$ order.

Definition 4 (approximate subdivision). An approximate subdivision is a set of curves with a binary relation, denoted $\prec_{y}$, whose transitive closure is irreflexive, such that $e \prec_{y} f$ or $f \prec_{y} e$ iff $e$ and $f$ overlap in $x$. The notation $e \preceq_{y} f$ means $e \prec_{y} f$ or $e=f, e \succ_{y} f$ means $f \prec_{y} e$, and $e \succeq_{y} f$ means $f \preceq_{y} e$.

Fig. 3 a shows an example in which the $\prec_{y}$ order for curves that overlap in $x$ is index order, e.g. $e_{3} \prec_{y} e_{5}$ because $3<5$. Although $\prec_{y}$ agrees with $<_{y}$ here, they disagree elsewhere. For example, $e_{2}$ intersects $e_{5}$, while $e_{9} \prec_{y} e_{10}$ yet $e_{10}<_{y} e_{9}$.

An approximate subdivision (Fig. 3 3 ) has a realization when an endpointpreserving deformation (Fig. 3 $\mathrm{b}$ ) of the curves can make the actual $<_{y}$ order agree with the imposed $\prec_{y}$ order (Def. [5). A realizable approximate subdivision represents an infinite number of shapes, but their curves all have the same endpoints and $<_{y}$ order, hence all these shapes have the same combinatorial structure. An unrealizable approximate subdivision does not represent any shape. 


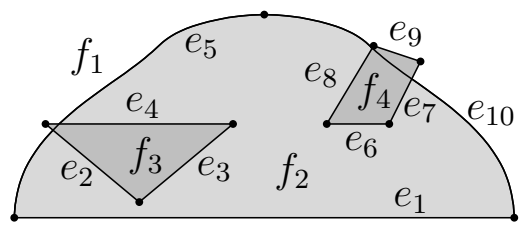

(a)

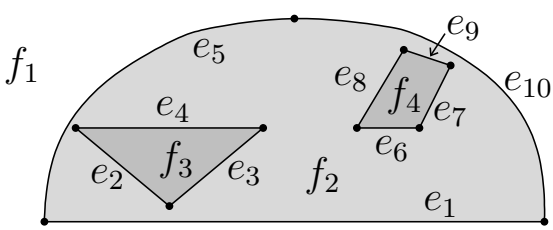

(b)

Figure 3: Approximate subdivision (a) and realization (b).

Definition 5 (realization). A realization of an approximate subdivision, $S$, is a function, $r$, from $S$ onto a subdivision, $T$, such that for all $e \in S$, e and $r(e)$ have the same endpoints and for all $e, f \in S, e \prec_{y} f$ implies $r(e)<_{y} r(f)$.

As indicated in the introduction, the vertical order of an endpoint, $p$, of a curve, $f$, with respect to a curve $e$, can be deduced from the order of $e$ and $f$. In this manner, we define the lower and upper sets of endpoints, $L(e)$ and $U(e)$, with respect to $e$ (Def. 6), from the imposed vertical order $\prec_{y}$. We use these sets to define four consistency constraints (Def. 7). Our central theorem is that an approximate subdivision has a realization iff it satisfies these four consistency constraints (Thm. 3).

Definition $6(L(e)$ and $U(e))$. For a curve, $e[a, b] \in S$, an approximate subdivision, $L(e)$ is the set of endpoints, $p$, of curves, $f \preceq_{y} e$, with $a_{x} \leq p_{x} \leq b_{x}$; likewise, $U(e)$ with $f \succeq_{y} e$.

Definition 7 (consistent). An approximate subdivision, $S$, is consistent if each curve, $e[a, b] \in S$, satisfies four constraints.

1. For each $q \in U(e)$, there is no $p \in L(e)$ with $a_{x}<p_{x}=q_{x}<b_{x}$ and $p_{y} \geq q_{y}$.

2. For each $f[c, d] \succ_{y} e, a_{x}=c_{x}$ implies $a_{y} \leq c_{y}$ and $b_{x}=d_{x}$ implies $b_{y} \leq d_{y}$.

3. a) For each $p \in L(e)$, there are no $c, d \in U(e)$ with $c_{x}<p_{x}<d_{x}$ and $c_{y}, d_{y} \leq p_{y}$. b) For each $q \in U(e)$, there are no $c, d \in L(e)$ with $c_{x}<q_{x}<$ $d_{x}$ and $c_{y}, d_{y} \geq q_{y}$.

4. If $e$ is vertical, there is no endpoint, $p$, of curve $f$, with $p_{x}=a_{x}$ and $p_{y} \in\left(a_{y}, b_{y}\right)$, the open interval between $a_{y}$ and $b_{y}$.

Fig. 目 illustrates the constraints. Part (a) violates constraint 1 if $e \prec_{y} g$, $f \prec_{y} e, p_{x}=q_{x}$, and $p_{y} \geq q_{y}$. (Fig. 2a is a special case with $e=e_{2}, f=e_{3}$, $g=e_{1}$ and $p=q=b$.) Part (b) violates constraint 2 if $e \prec_{y} f, a_{x}=c_{x}$, and $a_{y}>c_{y}$. Part (c) violates constraint 3a if $f \prec_{y} e, e \prec_{y} g, e \prec_{y} h, c_{x}<p_{x}<d_{x}$, and $p_{y}>c_{y}, d_{y}$. Part (d) violates constraint 4 if $a_{x}=p_{x}=b_{x}$ and $a_{y}<p_{y}<b_{y}$. All four of these violations occur in practice (Sec. 8). 


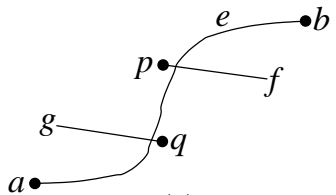

(a)

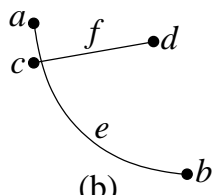

(b)
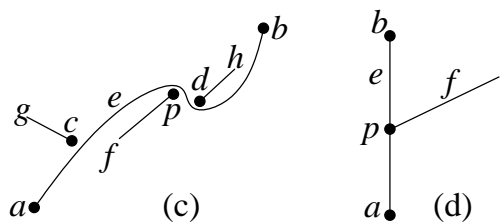

Figure 4: Inconsistent approximate subdivisions.

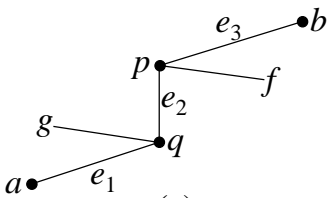

(a)

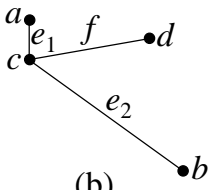

(b)
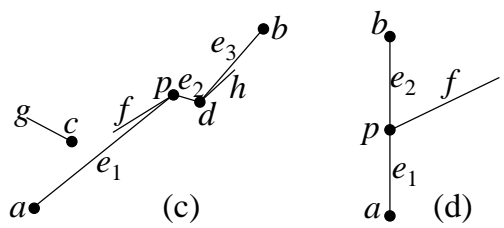

Figure 5: Refinements of the approximate subdivisions in Fig. 4.

Theorem 3. An approximate subdivision is realizable iff it is consistent (p. 17).

A refinement of an approximate subdivision is an approximate subdivision whose curves are sub-curves of the original curves with the induced $\prec_{y}$ order (Def. 9).

Definition 8 (refinement of curve). A refinement of a curve, $e[a, b]$, is a set of curves, $C(e)$, that form an $x$-monotone chain from $a$ to $b$.

Definition 9 (refinement of approximate subdivision). A refinement of an approximate subdivision, $S$, is an approximate subdivision, $S^{\prime}$, with the same set of endpoints in which each curve, $e \in S$, maps to a refinement, $C(e) \subset S^{\prime}$, and $e \prec_{y} f$ in $S$ implies $e^{\prime} \prec_{y} f^{\prime}$ in $S^{\prime}$ for every $e^{\prime} \in C(e)$ and $f^{\prime} \in C(f)$ that overlap in $x$.

Fig. 5 shows consistent refinements of the inconsistent approximate subdivisions from Fig. 团with $C(e)=\left\{e_{1}, e_{2}, e_{3}\right\}$ in parts (a) and (c) and $C(e)=\left\{e_{1}, e_{2}\right\}$ in parts (b) and (d). The refinements are realizations, except in part (c) where $e_{1}<_{y} f$ but $f \prec_{y} e$, and $h<_{y} e_{3}$ but $e \prec_{y} h$. However, a realization exists by Thm. 3 ,

Theorem 1. The refinement algorithm computes a consistent refinement (p. 12).

The remaining concepts relate to error analysis. We define an error metric on approximate subdivisions that models errors in the pairwise $\prec_{y}$ orders due to errors in the geometric primitives used to compute them, especially curve intersection point computation. An approximate subdivision is $\delta$-accurate when the $\prec_{y}$ order of each pair of curves equals the $<_{y}$ order of a pair of $\delta$-close curves (Def. 111). Each pair of curves can be placed in $\prec_{y}$ order by a small deformation.

Definition 10. $A$-deformation of a curve, $e$, is an $x$-monotone curve with the same endpoints as e whose Hausdorff distance from $e$ is less than $\delta$. 


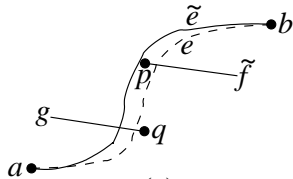

(a)

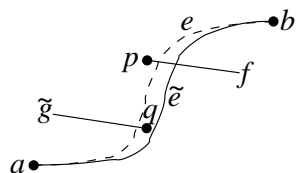

(b)

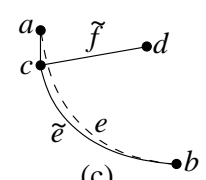

(c)

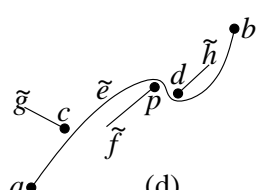

(d)

Figure 6: Illustrations of $\delta$-accuracy.

Definition 11 ( $\delta$-accurate). An approximate subdivision is $\delta$-accurate if $e \prec_{y} f$ implies that e and $f$ have $\delta$-deformations, $\tilde{e}$ and $\tilde{f}$, with $\tilde{e}<_{y} \tilde{f}$.

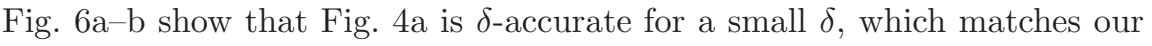
intuition that the curves barely intersect. The pairs $e, f$ and $e, g$ use different $\tilde{e}$ curves. Fig. 6 r shows that Fig. 4 b is $\delta$-accurate. Even though $c \in \tilde{e}, \tilde{e}<_{y} \tilde{f}$ because curves are open. Fig. 6 d shows that Fig. 迎 is $\delta$-accurate. The curves are their own $\delta$-perturbations, and $\tilde{e}$ is $x$-monotone but not monotone. Fig. 四 $\mathrm{d}$ is $\delta$-accurate because $e$ and $f$ do not overlap in $x$.

A refinement is a $\delta$-splitting when every split point is $\delta$-close to the curves

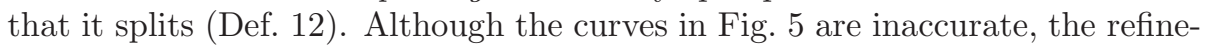
ment is a $\delta$-splitting because $e$ is split at points that are $\delta$-close to it.

Definition 12 ( $\delta$-splitting). A refinement of an approximate subdivision, $S$, is $a \delta$-splitting if for all $e \in S$ and $e^{\prime}\left[a^{\prime}, b^{\prime}\right] \in C(e)$, dist $\left(a^{\prime}, e\right)$, dist $\left(b^{\prime}, e\right)<\delta$, where $\operatorname{dist}(a, e)$ denotes the Hausdorff distance from a point, a, to a curve, e.

Lemma 15. The refinement algorithm output is a $\delta$-splitting (p. 201).

The output error of the refinement algorithm is due to the input error and the refinement error. If the input is $\delta$-accurate and the refinement is a $\delta$-splitting, the output is $\delta$-accurate. A stronger error bound is that the output has a realization in which each curve is $\delta$-close to its preimage curve, called a $\delta$ refinement (Def. 13). A $\delta$-refinement has a single $\delta$-deformation that places all the curves in $\prec_{y}$ order, whereas $\delta$-accuracy allows a separate $\delta$-deformation for each pair of curves. The realizations in Fig. 7 show that the refinements in Fig. 5 are $\delta$-refinements.

Definition 13 ( $\delta$-refinement). A $\delta$-refinement of an approximate subdivision, $S$, is a refinement that has a realization such that the Hausdorff distance from $r\left(e^{\prime}\right)$ to $e$ is bounded by $\delta$ for all $e \in S$ and $e^{\prime} \in C(e)$.

We conclude the error analysis by proving (Sec. 55) that the stronger error bound is implied by the weaker one and consistency, hence that the refinement algorithm computes a $\delta$-refinement of its input.

Theorem 5. A consistent $\delta$-splitting of a $\delta$-accurate approximate subdivision is a $\delta$-refinement ( $p$. 201).

Theorem 6. The refinement algorithm computes a $\delta$-refinement ( $p$. 211). 


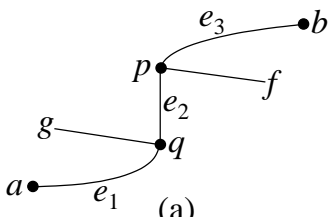

(a)

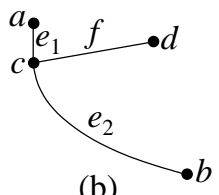

(b)

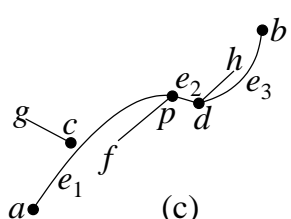

(c)

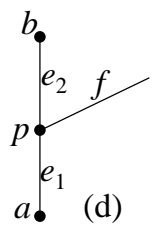

(d)

Figure 7: Realizations of Fig. 5 that are $\delta$-close to the approximate subdivisions in Fig. 4.

\section{Refinement Algorithm}

The refinement algorithm consists of four steps that enforce the four constraints in Def. 7 by splitting curves at endpoints of other curves. Splitting $e[t, h]$ at $p$ replaces $e$ by a refinement, $\left\{e_{1}[t, p], e_{2}[p, h]\right\}$, such that $e_{i} \prec_{y} f$ (or $e_{i} \succ_{y} f$ ) iff $e \prec_{y} f$ (or $e \succ_{y} f$ ) and $e_{i}$ and $f$ overlap in $x$. For simplicity, one can choose $e_{1}[t, p]$ and $e_{2}[p, h]$ to be the line segments $t p$ and $p h$. Even though this may be inaccurate, the ultimate realization of the refinement will be close to the original curves (Thm. 酐.

In the following description, a curve, $e[t, h]$, is classified with respect to an endpoint coordinate, $x$, as follows: $t_{x}=x=h_{x}$, vertical; $t_{x}=x<h_{x}$, outgoing; $t_{x}<x=h_{x}$, incoming; and $t_{x}<x<h_{x}$, passing.

Step 1 We begin with an example using curves $f_{1}$ to $f_{10}$ and $\prec_{y}$ equal to index order for curves that overlap in $x$ (Fig. 8 a). Because $f_{7} \prec_{y} f_{9}, w \in L\left(f_{9}\right)$, yet because $f_{9} \prec_{y} f_{10}, w \in U\left(f_{9}\right)$. Hence, curve $f_{9}$ and endpoint $w$ violate constraint 1 if we set $e=f_{9}, p=w$, and $q=w$. The algorithm visits, in an order consistent with $\prec_{y}$, the curves that have an endpoint whose $x$-coordinate equals $w_{x}$ : $f_{1}, f_{2}, f_{3}, f_{4}, f_{5}, f_{7}, f_{8}$, and $f_{10}$. (Curve $f_{4}[u, v]$ is vertical and $u_{x}=v_{x}=w_{x}$.) It forms groups of these curves separated by passing curves: $G_{1}=\left\{f_{1}, f_{2}, f_{3}, f_{4}, f_{5}\right\}$, passing curve $f_{6}, G_{2}=\left\{f_{7}, f_{8}\right\}$, passing curve $f_{9}$, and $G_{3}=\left\{f_{10}\right\}$. Each curve is added to the latest group and the constraint is checked. The check fails when the highest endpoint, $p$, in the previous group and the lowest endpoint, $q$, in the current group satisfy $p_{y} \geq q_{y}$. The algorithm splits at $p$ each passing curve $e$ that separate the previous group from the current group (because $e, p$, and $q$ violate constraint 1), and it then merges these two groups and the split curves into a single group. In our example, the check fails after $f_{10}$ is added to $G_{3}, f_{9}$ is split at $w$ into $f_{9}^{\prime}$ and $f_{9}^{\prime \prime}, G_{3}$ is merged into $G_{2}$, and the algorithm is done (Fig. 8 $\mathrm{b}$ ).

The algorithm is a plane sweep with a vertical sweep line. The sweep list, $L$, consists of curves in $\prec_{y}$ order. The events are the endpoint $x$-coordinates in increasing order. The incoming curves at $x$ are removed from $L$, the non-passing curves are handled in $\prec_{y}$ order as described below, and the outgoing curves are inserted into $L$. We write $f \prec_{y} G$ or $G \prec_{y} f$ when $f \prec_{y} g$ or $g \prec_{y} f$ for every $g$ in group $G$. The endpoints, $v$, of curves in $G$ with $v_{x}=x$ and with minimum 

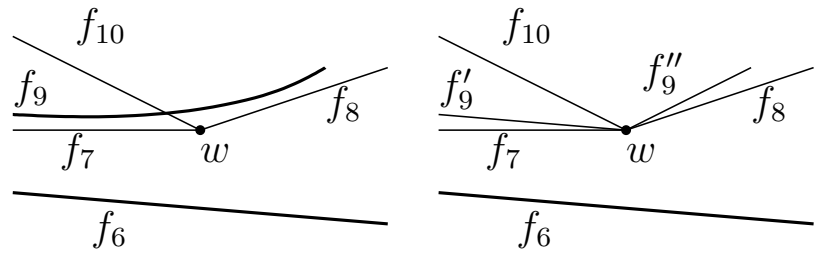

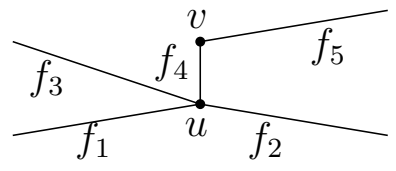

(a)

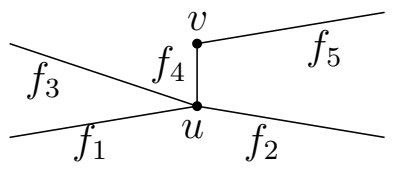

(b)

Figure 8: Curves before (a) and after (b) constraint 1 enforcement.

and maximum $y$-coordinates are denoted $\operatorname{ymin}(G)$ and $\operatorname{ymax}(G)$. A curve, $e$, is handled as follows.

1. If $m=0$ or there exists a passing $f$ with $G_{m} \prec_{y} f \prec_{y} e$

increment $m$ and set $G_{m}=\{e\}$

else add $e$ to $G_{m}$.

2. While $m>1$ and $\operatorname{ymin}\left(G_{m}\right)_{y} \leq \operatorname{ymax}\left(G_{m-1}\right)_{y}$ :

a. For each passing curve, $G_{m-1} \prec_{y} g \prec_{y} G_{m}$ :

i. Remove $g$ from $L$.

ii. Split $g$ into $g^{\prime}$ and $g^{\prime \prime}$ at $\operatorname{ymax}\left(G_{m-1}\right)$.

iii. Add $g^{\prime}$ and $g^{\prime \prime}$ to $G_{m-1}$.

b. Insert the curves of $G_{m}$ into $G_{m-1}$ and decrement $m$.

Step 2 We begin with an example using curves $f_{1}$ to $f_{4}$ that are incoming at $x=b_{1 x}=b_{2 x}=b_{3 x}=b_{4 x}$ and with $\prec_{y}$ equal to index order (Fig. 9 a). The pair $\left\langle f_{2}, f_{3}\right\rangle$ violates constraint 2 with $e=f_{2}, f=f_{3}, b=b_{2}$ and $d=b_{3}$, and similarly $\left\langle f_{1}, f_{3}\right\rangle$. We can handle $\left\langle f_{2}, f_{3}\right\rangle$ by splitting $f_{2}$ at $b_{3}$ or by splitting $f_{3}$ at $b_{2}$. The latter is more accurate because $\operatorname{dist}\left(b_{2}, f_{3}\right)$ (shown with a dashed line) is less than $\operatorname{dist}\left(b_{3}, f_{2}\right)$. We say that $b_{2}$ is safe for $\left\langle f_{2}, f_{3}\right\rangle$. Likewise, $b_{3}$ is safe for $\left\langle f_{1}, f_{3}\right\rangle$ if $\operatorname{dist}\left(b_{3}, f_{1}\right) \leq \operatorname{dist}\left(b_{1}, f_{3}\right)$. We remove the two violations by splitting $f_{1}$ into $\left\{f_{1}^{\prime}, b_{3} b_{1}\right\}$ and $f_{3}$ into $\left\{f_{3}^{\prime}, b_{2} b_{3}\right\}$ (Fig. 9] ).

The algorithm enforces constraint 2 via safe splits of curves that violate it. It processes the incoming curves at each endpoint $x$-coordinate as follows; outgoing curves are processed analogously. Let $s$ be the list of incoming curves in $\prec_{y}$ order. A safe endpoint for $e[v, a] \in s$ is an endpoint, $b$, of a curve, $f[w, b] \in s$, with $\operatorname{dist}(b, e) \leq \operatorname{dist}(a, f)$. All distances are measured with respect to the input curves: for a refinement curve $e^{\prime} \in C(e)$ (Def. 9), use $\operatorname{dist}(b, e)$ in place of $\operatorname{dist}\left(b, e^{\prime}\right)$. For endpoints $a$ and $b$, let $\min (a, b)$ denote $a$ if $a_{y}<b_{y}$ and $b$ otherwise; likewise $\max (a, b)$. Define $\mathrm{m}(e)$ as the minimum endpoint over $g \succeq_{y} e$ that is safe for $e$. In our example, $\mathrm{m}\left(f_{1}\right)=b_{3}, \mathrm{~m}\left(f_{2}\right)=b_{2}, \mathrm{~m}\left(f_{3}\right)=$ 


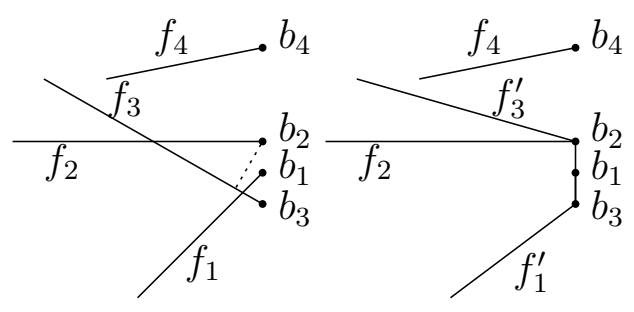

(a)

(b)

Figure 9: Curves before (a) and after (b) constraint 2 enforcement.

$b_{3}, \mathrm{~m}\left(f_{4}\right)=b_{4}$. The algorithm computes $\mathrm{m}(e)$ for every $e \in s$ then splits each curve, $e$, at the maximum $\mathrm{m}(f)$ over $f \preceq_{y} e$.

1. For each curve, $e[v, a]$, in $s$ :

a. Set $\mathrm{m}(e)=a$.

b. Append $e$ to $I$.

c. While $a_{y}<b_{y}$ for the predecessor, $f[w, b]$, of $e$ in $I$ :

i. Swap $e$ and $f$ in $I$.

ii. If $a$ is safe for $f$, set $\mathrm{m}(f)=\min (\mathrm{m}(f), a)$.

2. For each curve, $e$, in $s$

Split $e$ at the maximum of $\mathrm{m}(f)$ over $f \preceq_{y} e$ in $s$.

Steps 1-2 of the algorithm compute $\mathrm{m}(e)$ by inserting each curve, $e[v, a]$, into an initially empty list, $I$, ordered by endpoint $y$-coordinate. First, $e$ is appended to $I$ then it is swapped with each predecessor, $f[w, b]$, with $b_{y}>a_{y}$ until it reaches the correct position in $I$. In our example, $f_{3}$ is inserted into $I=\left(f_{1}, f_{2}\right)$ with two swaps after which $I=\left(f_{3}, f_{1}, f_{2}\right)$. Each swap reveals a constraint violation, hence implies a possible update to $\mathrm{m}\left(f_{3}\right)$.

Step 3 We begin with an example with curves $g[t, h], f_{1}\left[a_{1}, b_{1}\right]$ and $f_{2}\left[a_{2}, b_{2}\right]$ with $f_{1} \prec_{y} g$ and $g \prec_{y} f_{2}$ (Fig. 10 ). Curve $e=g$ and $p=a_{2} \in U(e)$ violate constraint $3 \mathrm{~b}$ with $c=t$, and $d=b_{1}$. The algorithm constructs a monotone curve from $t$ to $h$ that is above every $p \in L(g)$ and is below every $q \in U(g)$. It visits these endpoints in increasing $x$ order and tracks the rightmost one, $\mathrm{v}(g)$, where the curve is forced to increase or decrease. A constraint violation is detected when $\mathrm{v}(g)$ switches between increasing and decreasing, and $g$ is split at the previous $\mathrm{v}(g)$. Specifically, $\mathrm{v}(g)$ starts as $t$, is set to $a_{2}$, is unchanged at $b_{2}$ and $a_{1}$, is set to $b_{1}$, a violation is detected, and $g$ is split at $a_{2}$ (Fig. 10b).

The algorithm is a sweep with $L$ as before. Each $e \in L$ stores an endpoint, $\mathrm{v}(e)$, and a flag, $\operatorname{vtype}(e)$, that indicates if $\mathrm{v}(e)$ is on, above, or below $e$. Each input curve, $f[t, h]$, has a tail event at $t_{x}$ and a head event at $h_{x}$. The events are handled in increasing $x$ order with ties broken as follows: heads of non-vertical curves, tails of vertical curves, heads of vertical curves, and tails of non-vertical curves. 


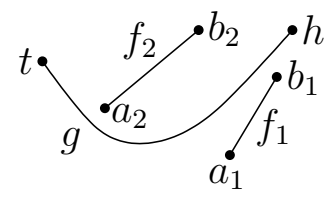

(a)

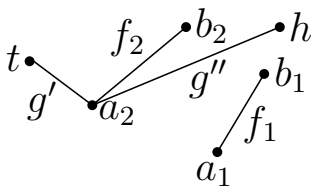

(b)

Figure 10: Curves before (a) and after (b) constraint 3 enforcement.

tail event for $f[t, h]$ :

1. If $f$ is not vertical, insert it into $L$ with $\mathrm{v}(f)=t$ and vtype $(f)=$ on.

2. For each $e \in L$ with $e \prec_{y} f$ and $t_{y} \leq \mathrm{v}(e)_{y}$, call lower $(e, t)$.

3. For each $g \in L$ with $g \succ_{y} f$ and $t_{y} \geq \mathrm{v}(g)_{y}$, call raise $(g, t)$.

head event for $f[t, h]$ :

1. For each $e \in L$ with $e \preceq_{y} f$ and $h_{y} \leq \mathrm{v}(e)_{y}$, call lower $(e, h)$.

2. For each $g \in L$ with $g \succeq_{y} f$ and $h_{y} \geq \mathrm{v}(g)_{y}$, call raise $(g, h)$.

3. If $f$ is not vertical, remove it from $L$.

subroutine lower $(e, a)$ :

1. If $\operatorname{vtype}(e)=$ below, split $e$ at $\mathrm{v}(e)$.

2 . Set $\mathrm{v}(e)=a$ and $\operatorname{vtype}(e)=$ above.

subroutine raise $(e, a)$ :

1. If $\operatorname{vtype}(e)=$ above, split $e$ at $\mathrm{v}(e)$.

2. Set $\mathrm{v}(e)=a$ and $\operatorname{vtype}(e)=$ below.

This algorithm handles strict extrema. In the general case, lower sets a pointer from $a$ to $\mathrm{v}(e)$ if $\operatorname{vtype}(e)=$ above and $\mathrm{v}(e)_{y}=a_{y}$; likewise if $\operatorname{vtype}(e)=$ below in raise. The split steps traverse the pointers and split $e$ at the extrema.

Step 4 Split every vertical curve at every endpoint that violates constraint 4.

\section{Correctness}

We prove that the refinement algorithm output is a consistent refinement of the input (Thm. 1) and that the algorithm is inconsistency sensitive (Thm. 2). We then prove that an approximate subdivision is consistent iff it has a realization (Thm. 3), which implies that the refinement algorithm output has a realization.

Theorem 1. The refinement algorithm computes a consistent refinement.

Proof. We prove consistency. Being a refinement follows from the definition of a curve split.

Step 1 enforces the invariant that constraint 1 holds for every passing curve, $f$, and the endpoints in $G_{1}, \ldots, G_{m}$. Step 2 enforces constraint 2 by assigning 
each incoming curve an endpoint whose $y$-coordinate is the maximum over all incoming curves below it in $\prec_{y}$ order. For step 3 , let input curve $e$ and endpoint $q \in U(e)$ violate constraint $3 \mathrm{~b}$ with $c, d \in L(e)$; the other case is similar. If $e$ is split at an endpoint, $w$, with $c_{x}<w_{x}<d_{x}$ before $d$ is handled, we are done. Otherwise, $\mathrm{v}(e)_{y} \geq c_{y}$ after $c$ is handled, so $\mathrm{v}(e)_{y} \leq q_{y}$ and vtype $(e)=$ above after $q$ is handled. When $d$ is handled, vtype $(e)=$ above and $\mathrm{v}(e)=r$ for some $r \in U(e)$ with $r_{y} \leq q_{y}$ and $c_{x}<r_{x}<d_{x}$, since no split occurs before $d$. Thus, $e$ is split at $r$, which eliminates the $e, q$ inconsistency. Step 4 trivially enforces constraint 4.

Step 2 maintains constraint 1 by not changing $L(e)$ or $U(e)$ for a passing curve, $e$. Step 3 maintains constraint 1 by not adding an endpoint, $v$, to $U(e)$, except by splitting $e$ at $v$ (proof below). It maintains constraint 2 by splitting a curve, $e$, at the endpoint $v \in L(e)$ or $v \in U(e)$ with the largest or smallest $v_{y}$ value. Step 4 maintains constraints $1-3$ by only splitting vertical curves.

Proof: Suppose $e \prec f, v \in L(e) \cap L(f)$, and $f$ is split at $v$. We show that $e$ is also split at $v$. After $v$ is processed, $\mathrm{v}(f)=v$, since it is later split at $v$. By Lem. 11below, $\mathrm{v}(e)_{y} \leq v_{y}$. But $\mathrm{v}(e)_{y} \geq v_{y}$ because $v \in L(e)$, so $\mathrm{v}(e)=v$. Curve $f$ is split at $v$ when the sweep processes an endpoint, $u \in U(f)$ with $u_{y} \leq v_{y}$. If $\mathrm{v}(e)=v$ then, $e$ is split at $v$ because $u \in U(e)$. Otherwise, $e$ is lowered by $w \in U(e) \cap L(f)$ with $w_{x}<u_{x}$ and $e$ is split at $v$ then.

Lemma 1. In step 3, $\mathrm{v}(e)_{y} \leq \mathrm{v}(f)_{y}$ for $e, f \in L$ with $e \prec_{y} f$.

Proof. The invariant holds initially and is preserved by sweep updates because if $v \in L(e)$ overlaps $f$ in $x$, then $v \in L(f)$. If we call raise $(e, v)$ and if $v_{y} \geq$ $\mathrm{v}(f)_{y}$, then we also call raise $(f, v)$. Hence $\mathrm{v}(e)_{y} \leq \mathrm{v}(f)_{y}$ remains true because $\mathrm{v}(e)_{y}=\mathrm{v}(f)_{y}=v$. Similarly for $u \in U(f)$.

To prove inconsistency sensitivity, we need to count constraint violations. A constraint 1 violation is a pair $e, q$ such that there exists $p$ that violates the constraint, and similarly the other violations are defined by the pair $e, f(2)$, the pair $e, p(3 \mathrm{a})$, the pair $e, q(3 \mathrm{~b})$, or the pair $e, p(4)$.

Theorem 2. The refinement algorithm complexity is $\mathrm{O}(n \log n+k)$ for $n$ curves with $k=\mathrm{O}\left(n^{2}\right)$ constraint violations.

Proof. The definitions of the constraint violations imply that $k=\mathrm{O}\left(n^{2}\right)$, since there are $n$ curves and $\mathrm{O}(n)$ endpoints. Each curve, $e$, stores its poset height, height $(e)$. For $e$ and $f$ that overlap in $x, e \prec_{y} f$ iff height $(e)<\operatorname{height}(f)$. Each curve stores pointers to the curves below and above its endpoints. Splitting a curve updates these pointers in constant time. Sort the endpoints in $x$ order with ties broken by height. This processing takes $\mathrm{O}(n \log n)$ time and $\mathrm{O}(n)$ space.

Step 1 takes $\mathrm{O}(n+k)$ time using a doubly linked list to represent $L$. Group operations take constant time using ymin, ymax, and the maximum curve height, hmax, to represent a group.

Step 2 takes $\mathrm{O}(n+k)$ time because the number of constraint 2 violations is $\mathrm{O}(k)$ after step 1 , which is shown as follows. If step 1 splits $e$ at $p, p$ is the 
highest point in $G_{m-1}$, so the new curves satisfy constraint 2 with respect to a curve, $f \prec_{y} e$. A constraint 2 violation is created for each curve, $f \succ_{y} e$, that has an endpoint, $q$, with $q_{x}=p_{x}$ and $q_{y}<p_{y}$. Since $q \in U(e), e$ and $f$ are also a constraint 1 violation.

Step 3 takes $\mathrm{O}(n \log n+k)$ time. Maintain a partition of $L$ into sublists with a common $\mathrm{v}$ and vtype. Each sublist stores pointers to its lowest and highest curves. Represent the partition as a balanced binary tree of sublists ordered by $\mathrm{v}_{y}$. By Lem. 1, these will also be ordered by $h$. Store the curves in $L$ in a binary tree ordered by $h$. To handle an event, look up the new curve in the latter binary tree to determine the $h$ of its neighbors. Look up this $h$ in the former binary tree to determine the sublist that will be split. The new curve can lower the curves below it in its sublist or can raise the curves above. Each case splits the sublist into lower and upper sublists. When a lower or a raise propagates to sublists below or above, they are merged into the newly created lower or upper sublists. A tail event also creates a singleton sublist for the curve that enters $L$. A head event removes its curve from its sublist and removes an empty sublist from the partition.

Excluding sublist merges and curve splits, the sweep takes $\mathrm{O}(n \log n)$ because there are $\mathrm{O}(n)$ tree operations. Although an event can cause $\mathrm{O}(n)$ merges, the merge time is $\mathrm{O}(n \log n)$ because each merge decreases the number of sublists, whereas at most $2 n$ sublists are created. Since each split applies to an entire sublist, the partition structure is unchanged and the only cost is $\mathrm{O}(k)$ to update the curves.

Step 4 takes $\mathrm{O}(n+k)$ time, using the sorted endpoints, because the number of constraint 4 violations is $\mathrm{O}(k)$ after steps $1-3$. Each split creates at most one violation and is charged to a constraint $1-3$ violation.

We now turn to Thm. 3. We first prove necessity (Lem. 2). To prove sufficiency, we start with a more general sufficient condition (Def. 15] and Lem. 33).

Lemma 2. If an approximate subdivision has a realization, it is consistent.

Proof. For constraint 1, consider $p \in L(e)$ and $q \in U(e)$ with $a_{x}<p_{x}=q_{x}<b_{x}$ (Fig. 4 ). There exists a curve $f \prec_{y} e$ with endpoint $p$ and a curve $g \succ_{y} e$ with endpoint $q$. Hence, $r(f)<_{y} r(e)$ and $r(e)<_{y} r(g)$. By continuity, $p<_{y} \overline{r(e)}$ or $p \in \overline{r(e)}$, but $r(e) \cap \overline{r(f)}=\emptyset$ by Def. 5 and $a_{x}<p_{x}<b_{x}$, hence $p<_{y} r(e)$. Similarly, $r(e)<_{y} q$ and hence $p_{y}<q_{y}$.

For constraint 2, consider $a_{x}=c_{x}$ (Fig. 4b). Since $r(e)<_{y} r(f), a \in \overline{r(e)}$, and $c \in \overline{r(f)}, a_{y} \leq c_{y}$. The $b_{x}=d_{x}$ case is similar.

For constraint $3 \mathrm{a}$, consider $e[a, b]$ increasing (Fig. 4 $)$. As above, $p \in L(e)$ and $a_{x}<p_{x}<b_{x}$ imply $p<_{y} r(e)$, and $d \in U(e)$ and $a_{x}<d_{x}$ imply $r(e)<_{y} d$ or $d=b$. Since $r(e)$ is monotone and $p_{x}<d_{x}, p_{y}<d_{y}$. Similarly, $p_{y}<c_{y}$ for $e$ horizontal or decreasing. The constraint $3 \mathrm{~b}$ proof is similar.

Constraint 4 holds because $r(e) \cap \overline{r(f)}=\emptyset$ by Def. 5 (Fig. 4d). 


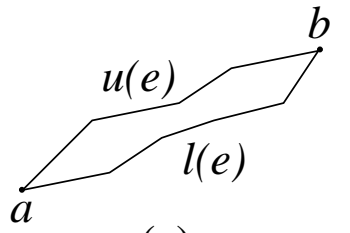

(a)

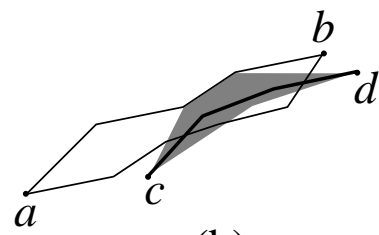

(b)

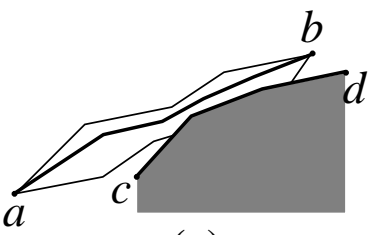

(c)

Figure 11: (a) $M(e)$; (b) $M(f)$ (shaded) and $r(f)$ (dark); (c) $M^{\prime}(e), r(e)$ (dark), and $r(f)^{-}$(shaded).

Definition 14 (monotone). A set, $M(e)$, is monotone with respect to a curve, $e[a, b]$, if $M(e)=e$ for $e$ horizontal or vertical and otherwise $M(e)$ is an open set bounded by monotone curves, $l(e)$ and $u(e)$, that connect a to b (Fig. 11a).

Definition 15 (ordered monotone assignment). An assignment of a monotone set, $M(e)$, to each curve, $e$, in an approximate subdivision is ordered if no $M(e)$ contains a curve endpoint, and $f \prec_{y} e$ implies $M(f)<_{y} M(e)$ unless $e$ and $f$ are both increasing or both decreasing in which case $M(f)<_{y} u(e)$ (Fig. 11b).

Definition $16\left(p^{-}, p^{+}, S^{-}\right.$and $\left.S^{+}\right)$. For a point, $p, p^{-}=\left\{\left(p_{x}, y\right) \mid y \leq p_{y}\right\}$ and $p^{+}=\left\{\left(p_{x}, y\right) \mid y \geq p_{y}\right\}$. For a set, $S, S^{-}=\bigcup_{p \in S} p^{-}$and $S^{+}=\bigcup_{p \in S} p^{+}$.

Lemma 3. If an approximate subdivision has an ordered monotone assignment, it has a realization.

Proof. For each curve, $e$, we will choose $r(e) \subset M(e)$ that will be disjoint from the other curve endpoints. If $e$ is horizontal or vertical, define $r(e)=M(e)=e$. Vertical $r(e)$ and $r(f)$ cannot intersect because they cannot contain each other's endpoints. Otherwise, $f \prec_{y} e$ implies $r(f)<_{y} r(e)$ because $M(f)<_{y} M(e)$, except when both are increasing or both are decreasing.

We define $m$ for the increasing curves in an order that is consistent with $\prec_{y}$. Let $M^{\prime}(e)$ equal $M(e)$ minus $r(f)^{-}$for all increasing $f \prec_{y} e$ (Fig.11k). Because $r(f) \subset M(f)<_{y} u(e)$ (Fig. 111), $M^{\prime}(e)$ is connected. Since the endpoints of $r(f)$ do not lie in $R(e)$, the only part of the boundary of $r(f)^{-}$that can lie in $R(e)$ is $r(f)$, hence the lower boundary of $M^{\prime}(e)$ is monotone. Choose a monotone path, $r(e) \in M^{\prime}(e)$, from $a$ to $b$ (Fig. 11k). Because $r(e) \cap r(f)^{-} \subset M^{\prime}(e) \cap r(f)^{-}=\emptyset$, $r(f)<_{y} r(e)$ as required. We handle the decreasing curves similarly.

We complete the sufficiency proof by showing that a consistent approximate subdivision has an ordered monotone assignment, $R(e)$.

Definition 17. For a point, $p, p^{++}=\left[p_{x}, \infty\right) \times\left[p_{y}, \infty\right), p^{-+}=\left(-\infty, p_{x}\right] \times$ $\left[p_{y}, \infty\right), p^{+-}=\left[p_{x}, \infty\right) \times\left(-\infty, p_{y}\right]$, and $p^{--}=\left(-\infty, p_{x}\right] \times\left(-\infty, p_{y}\right]$. For a set, $A, A^{++}=\bigcup_{p \in A} p^{++}$etc. For two points, $p$ and $q$, with $p_{x}<q_{x}, A(p, q)=$ $\left(p_{x}, q_{x}\right) \times\left(p_{y}, q_{y}\right)$. 
Definition $18(R(e))$. For a curve $e[a, b]$ :

$$
R(e)= \begin{cases}A(a, b)-L(e)^{+-} \cup U(e)^{-+} & \text {for increasing } e, \\ A(a, b)-L(e)^{--} \cup U(e)^{++} & \text {for decreasing } e, \\ e & \text { otherwise }\end{cases}
$$

Lemmas 4 through 7 apply to a consistent approximate subdivision.

Lemma 4. For a curve, $e[a, b]$, if $p \in L(e), q \in U(e)$, and either $p \notin\{a, b\}$ or $q \notin\{a, b\}$, e increasing or horizontal implies $p^{+-} \cap q^{-+}=\emptyset$ and e decreasing or horizontal implies $p^{--} \cap q^{++}=\emptyset$.

Proof. Suppose $e^{\prime}\left[a^{\prime}, b^{\prime}\right]$ is increasing or horizontal, $f^{\prime} \prec_{y} e^{\prime}$ has endpoint $p^{\prime} \in$ $L\left(e^{\prime}\right), g^{\prime} \succ_{y} e^{\prime}$ has endpoint $q^{\prime} \in U\left(e^{\prime}\right), q^{\prime} \notin\left\{a^{\prime}, b^{\prime}\right\}$, and $p^{{ }^{+-}} \cap q^{{ }^{-+}} \neq \emptyset$, hence $p_{x}^{\prime} \leq q_{x}^{\prime}$ and $p_{y}^{\prime} \geq q_{y}^{\prime}$. We will show a contradiction. The other cases are similar.

If $a_{x}^{\prime}<p_{x}^{\prime}=q_{x}^{\prime}<b_{x}^{\prime}$, constraint 1 is violated with $e[a, b]=e^{\prime}\left[a^{\prime}, b^{\prime}\right], p=p^{\prime}$, and $q=q^{\prime}$. If $a_{x}^{\prime}=p_{x}^{\prime}=q_{x}^{\prime}, p_{y}^{\prime} \leq a_{y}^{\prime}$ by constraint 2 with $e=f^{\prime}, f=e^{\prime}, a=p^{\prime}$, and $c=a^{\prime}$. Similarly, $a_{y}^{\prime} \leq q_{y}^{\prime}$, so $a_{y}^{\prime} \leq q_{y}^{\prime} \leq p_{y}^{\prime} \leq a_{y}^{\prime}$ and $q^{\prime}=a^{\prime}$, which is a contradiction. Similarly, $p_{x}^{\prime}=q_{x}^{\prime}=b_{x}^{\prime}$ is contradictory. If $a_{x}^{\prime}<p_{x}^{\prime}<q_{x}^{\prime}<b_{x}^{\prime}$, either $p_{y}^{\prime} \geq a_{y}^{\prime}$ or $q_{y}^{\prime} \leq b_{y}^{\prime}$. The former contradicts constraint 3 a with $e=e^{\prime}$, $c=a, p=p^{\prime}$, and $d=q^{\prime}$, and the latter contradicts constraint 3b with $e=e^{\prime}$, $c=p, q=q^{\prime}$, and $d=b^{\prime}$. If $a_{x}^{\prime}=p_{x}^{\prime}<q_{x}^{\prime}<b_{x}^{\prime}, p_{y}^{\prime} \leq a_{y}^{\prime}$ by constraint 2 and constraint $3 \mathrm{~b}$ is contradicted as before. Similarly, $a_{x}^{\prime}<p_{x}^{\prime}<q_{x}^{\prime}=b_{x}^{\prime}$ is contradictory. If $a_{x}^{\prime}=p_{x}^{\prime}$ and $q_{x}^{\prime}=b_{x}^{\prime}, p_{y}^{\prime} \leq a_{y}^{\prime}$ and $b_{y}^{\prime} \leq q_{y}^{\prime}$ by constraint 2, so $b_{y}^{\prime} \leq q_{y}^{\prime} \leq p_{y}^{\prime} \leq a_{y}^{\prime} \leq b_{y}^{\prime}$ and $q^{\prime}=b^{\prime}$, which is contradictory.

Lemma 5. $R(e)$ is monotone with respect to $e$.

Proof. For increasing $e$, the boundary of $p^{+-}$is monotone, so the boundary of $L(e)^{+-}$is monotone because it is the upper envelope of a set of monotone curves. Similarly, the boundary of $U(e)^{-+}$is monotone. By Lem. 目

$$
L(e)^{+-} \cap U(e)^{-+}=\cup_{p \in L(e), q \in U(e)} p^{+-} \cap q^{-+}=\{a, b\} .
$$

Hence, $R(e)$ is an open set bounded below and above by the portions of the boundaries of $L(e)^{+-}$and $U(e)^{-+}$that connect $a$ to $b$. Decreasing $e$ is handled similarly and the other cases are trivial.

Lemma 6. For $e, f$, and $g$ that overlap in $x, e \prec_{y} f$ and $f \prec_{y} g$ implies $e \prec_{y} g$.

Proof. Since $e$ and $g$ overlap in $x, e \prec_{y} g$ or $g \prec_{y} e$, but the latter implies that $(e, e)$ is in the transitive closure of $\prec_{y}$, which contradicts Def. 4.

Lemma 7. The assignment of $R(e)$ to $e$ is ordered.

Proof. Verticals cannot contain endpoints by constraint 4. A horizontal, $e[a, b]$, cannot contain an endpoint, $p$, by constraint 3 with $c=a$ and $d=b$. Otherwise, $R(e)$ cannot contain $p$ because one of $p^{--}, p^{-+}, p^{+-}, p^{++}$has been removed.

If $e[a, b]$ is not decreasing and $f[c, d]$ is not increasing and $f \prec_{y} e$, either $a=c, a \in U(f)-\{c, d\}$, or $c \in L(e)-\{a, b\}$. If $a=c, e$ and $f$ cannot both be 


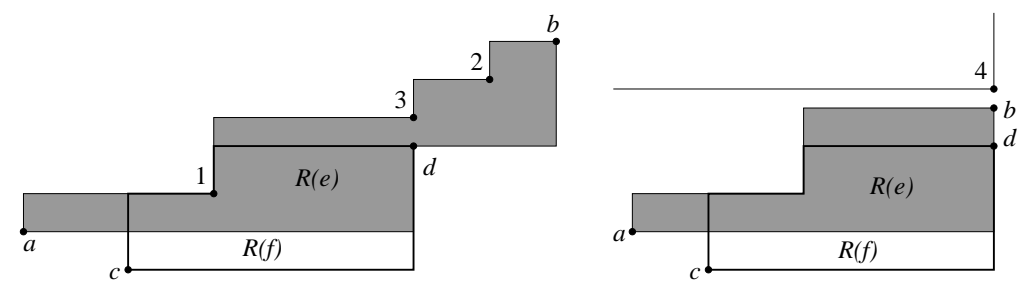

Figure 12: $R(e)$ is shaded and $R(f)$ has a thicker boundary.

horizontal without one containing the other's right endpoint. We have $p_{y} \leq a_{y}$ for $p \in R(f)$ and $q_{y} \geq a_{y}$ for $q \in R(e)$ with equality only for horizontal curves. Hence, $R(f)<_{y} R(e)$. If $a \in U(f)-\{c, d\}, R(f) \cap a^{++}=\emptyset$. Since $R(e) \subset a^{++}$, $R(f)<_{y} R(e)$. The $c \in L(e)-\{a, b\}$ case is similar, as is $e$ not increasing and $f$ not decreasing.

It remains to show that if $e[a, b]$ and $f[c, d]$ are both increasing and $f \prec_{y} e$, $R(f)<_{y} u(e)$, and similarly for both decreasing. It suffices to show $R(f)<_{y} q^{-+}$ for each $q \in U(e)$ for which $q^{-+}$and $f$ overlap in $x$. If $q_{x}<d_{x}$ (Fig. 12, $q=1$ ), a curve, $g \succ_{y} e$, with endpoint $q$ also overlaps $f$ in $x$, so $g \succ_{y} f$ by Lem. 6] $q \in U(f), q^{-+}$was subtracted from $R(f)$, and thus $R(f)<_{y} q^{-+}$. If $q_{x}>d_{x}$ (Fig. 12, $q=2), d \in L(e)$ because it is an endpoint of $f \prec_{y} e$. By Lem. 4 . $q^{-+} \cap d^{+-}=\emptyset$, hence $d_{y}<q_{y}$ and $R(f) \subset A(c, d)<_{y} q_{-+}$. If $q_{x}=d_{x}<b_{x}$ (Fig. 12, $q=3$ ), the same reasoning applies. If $q_{x}=d_{x}=b_{x}$ (Fig. 12 $q=4$ ), $q_{y} \geq b_{y}$ and $b_{y} \geq d_{y}$ by constraint 2 , so $R(f) \subset A(c, d)<_{y} q^{-+}$.

Theorem 3. An approximate subdivision has a realization iff it is consistent.

Proof. Lem. 2 proves necessity. Lems. 3 [5, and 7 prove sufficiency.

\section{Error Analysis}

We prove that a consistent $\delta$-splitting of a $\delta$-accurate approximate subdivision is a $\delta$-refinement (Thm. 5). This implies that the refinement algorithm output is a $\delta$-refinement (Thm. 6). We first prove the special case where the approximate subdivision is consistent, hence is its own refinement (Thm. (4). We define the $\delta$ offsets of a curve (Def. 19] and Fig. 13a) and prove them monotone (Lem. 9). We prove that the $\prec_{y}$ relation on the curves of a $\delta$-accurate approximate subdivision implies a $<_{y}$ relation on their $\delta$-offsets (Lems. 10 11). We use these tools to sharpen the Thm. 3 realization into one that is $\delta$-close to the approximate subdivision.

Lemma 8. For a point, a, and an increasing (decreasing) curve, e, $\operatorname{dist}(a, e)$ is decreasing (increasing) in $a_{x}$ and increasing in $a_{y}$ for $a>_{y} e$ and is increasing (decreasing) in $a_{x}$ and decreasing in $a_{y}$ for $a<_{y} e$.

Proof. Follows from Lem. 8 in our prior paper. [7] 


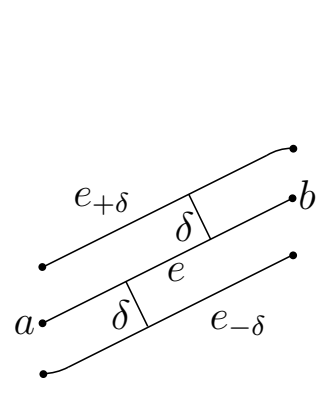

(a)

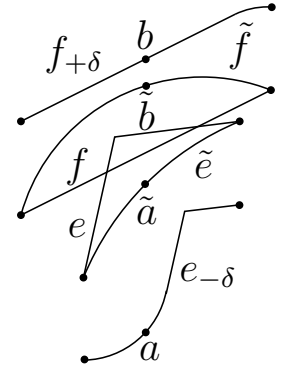

(b)

Figure 13: Offsets (a) and their properties (b).

Definition $19\left(e_{-\delta}\right.$ and $\left.e_{+\delta}\right)$. The $\delta$-offsets of a curve, $e$, are

$$
\begin{aligned}
& e_{-\delta}=\left\{a<_{y} e \mid \operatorname{dist}(a, e)=\delta\right\} \\
& e_{+\delta}=\left\{a>_{y} e \mid \operatorname{dist}(a, e)=\delta\right\} .
\end{aligned}
$$

Lemma 9. The offsets $e_{ \pm \delta}$ are monotone.

Proof. Let a vertical line intersect $e_{ \pm} \delta$ at $a$ and $b$ with $a_{y}<b_{y}$. Since $\operatorname{dist}(a, e)=$ $\delta$ and $\operatorname{dist}(b, e)=\delta \operatorname{dist}(c, e)=\delta$ for $c \in[a, b]$ by Lem. 8 . Hence, the line intersects $e_{ \pm} \delta$ in a connected set. Likewise for a horizontal line.

Lems. 10 and 11 apply to a $\delta$-accurate approximate subdivision.

Lemma 10. For two curves, $e$ and $f, e \preceq_{y} f$ implies $e_{-\delta}<_{y} f_{+\delta}$.

Proof. By Def. 19, $e_{-\delta}<_{y} e<_{y} e_{+\delta}$. For $e \prec_{y} f$, let $a \in e_{-\delta}$ and $b \in f_{+\delta}$ with $a_{x}=b_{x}$ (Fig. 13b). There exist $\delta$-deformations, $\tilde{e}$ and $\tilde{f}$, with $\tilde{e}<_{y} \tilde{f}$. Let $\tilde{a} \in \tilde{e}$ and $\tilde{b} \in \tilde{f}$ satisfy $\tilde{a}_{x}=\tilde{b}_{x}=a_{x}$, so $\tilde{a}_{y}<\tilde{b}_{y}$. We have $a_{y}<\tilde{a}_{y}$ by Lem. 8 because $\operatorname{dist}(a, e)=\delta$ and $\operatorname{dist}(\tilde{a}, e)<\delta$; likewise $\tilde{b}_{y}<b_{y}$. Thus, $a_{y}<\tilde{a}_{y}<\tilde{b}_{y}<b_{y}$.

Lemma 11. If $p \in L(e), p<_{y} \overline{e_{+}}$; if $q \in U(e), q>_{y} \overline{e_{-\delta}}$.

Proof. We prove the $p \in L(e)$ case for $e[a, b]$. By construction, $e<_{y} e_{+\delta}$ hence $\{a, b\}<_{y} \overline{e+\delta}$ by continuity and because $\operatorname{dist}(a, e)=\operatorname{dist}(b, e)=0<\delta$. If $\tilde{e}$ is a $\delta$-deformation of $e, \overline{\tilde{e}}=\tilde{e} \cup\{a, b\}<_{y} \overline{e_{+\delta}}$ and similarly $\overline{\tilde{e}}>_{y} \overline{e_{-\delta}}$.

If $p \in L(e), p$ is an endpoint of $f \prec_{y} e$ and hence an endpoint of $\tilde{f}<_{y} \tilde{e}$ by Def. 11. By continuity, $p_{y} \leq r_{y}$ for $r \in \overline{\tilde{e}}$ with $p_{x}=r_{x}$. Since $\overline{\tilde{e}}<_{y} \overline{e_{+\delta}}$, $r<_{y} \overline{e_{+} \delta}$ hence $p<_{y} \overline{e_{+} \delta}$.

For the proof of Thm. 4, $R_{\delta}(e)$ serves the role that $R(e)$ did for Thm. 3 ,

Definition $20\left(R_{\delta}(e)\right)$. For an approximate subdivision, define $R_{\delta}(e)=R(e)$ for horizontal and vertical e. For increasing e, $R_{\delta}(e)$ is $R(e)$ minus $f_{-\delta}^{-}$for all increasing $f \preceq_{y}$ e and minus $g_{+\delta}^{+}$for all increasing $g \succeq_{y}$ e (Fig. 14). Similarly for decreasing $e$. 


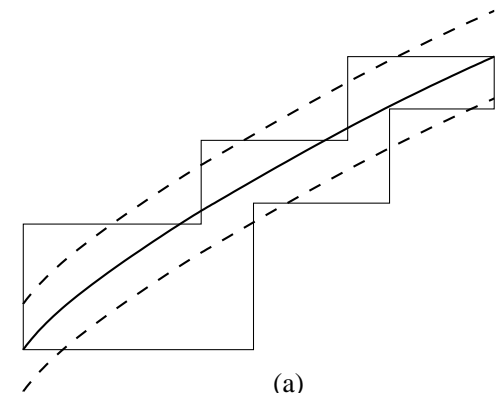

(a)

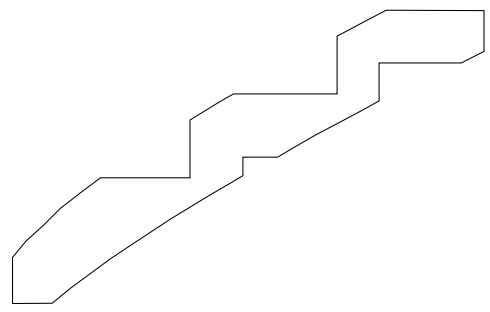

(b)

Figure 14: a) $e, e_{-\delta}, e_{+\delta}$, and $R(e)$; (b) $R(e)-e_{-\delta}^{-} \cup e_{+\delta}^{+}$.

Lemma 12. If an approximate subdivision is consistent and $\delta$-accurate, the sets $R_{\delta}(e)$ are monotone.

Proof. We prove the $e[a, b]$ increasing case. Let $f[c, d] \preceq_{y} e$ be increasing. For $q \in U(f), \overline{f_{-\delta}}<_{y} q$ by Lem. 11, so $f_{-\delta}<_{y} q^{-+}$, since $f_{-\delta}$ is increasing. Hence, $f_{-\delta}<_{y} U(f)^{-+}$, which implies $f_{-\delta} \subset R(f)^{-}$, since both span the $x$-interval $\left(c_{x}, d_{x}\right)$. Since $R(f)<_{y} u(e), R(f)^{-}<_{y} u(e)$ and $f_{-\delta}<_{y} u(e)$. Likewise, $g_{+\delta}>_{y} l(e)$ for increasing $g \succeq_{y} e$. If $f \preceq_{y} e, e \preceq_{y} g$, and $f$ and $g$ overlap in $x$, $f \preceq_{y} g$ by Lem. 6 and $f_{-\delta}<_{y} g_{+\delta}$ by Lem. 10, We conclude that the lower and upper sets that are removed from $R(e)$ are disjoint. Furthermore, $c, d \notin R(e)$ by Lem. 7 and $f_{-\delta}<_{y} f$. Thus, the only part of the boundary of $f_{-\delta}^{-}$that enters $R(e)$ is part of $f_{-\delta}$, which is monotonic. Therefore, the lower boundary of $R_{\delta}(e)$ is monotonic and similarly the upper boundary.

Lemma 13. Let $u_{\delta}(e)$ be the upper boundary of $R_{\delta}(e)$. If $f[c, d] \prec_{y} e[a, b]$ are both increasing or both decreasing, $R_{\delta}(f)<_{y} u_{\delta}(e)$.

Proof. Pick $p \in R_{\delta}(f)$ with $a_{x} \leq p_{x} \leq b_{x}$. Since $R_{\delta}(f) \subset R(f)<_{y} u(e)$, $p<_{y} u(e)$. Pick $g \succeq_{y} e$ that overlaps $p$ in $x$. Thus, $g$ overlaps $f$ in $x$ and $g \succ_{y} f$ by Lem. [6] Therefore, $g_{+\delta}^{+}$was removed from $R_{\delta}(f)$ hence $p<_{y} g_{+\delta}^{+}$. Since $p$ is below the upper sets removed from $R_{\delta}(e)$ that overlap it in $x, p<_{y} u_{\delta}(e)$. Hence, $R_{\delta}(f)<_{y} u_{\delta}(e)$.

Theorem 4. A consistent, $\delta$-accurate approximate subdivision is a $\delta$-refinement.

Proof. The $R_{\delta}(e)$ are monotone by Lem. 12, They are an ordered assignment because $R_{\delta}(e) \subset R(e)$, so $R_{\delta}(e)<_{y} R_{\delta}(f)$ for $e \prec_{y} f$ unless both are decreasing or both are increasing, and that case is covered by Lem. 13, By Lem. 3, there is a realization, $r(e) \subset R_{\delta}(e)$. For $p \in R_{\delta}(e)$, $\operatorname{dist}(p, e)<\delta$ by construction, so $r(e)$ is a $\delta$-deformation of $e$.

We prove Thm. 5 similarly, using Lem. 14 instead of Lem. 11 and $R_{\delta}^{\prime}\left(e^{\prime}\right)$ (Def. 21) instead of $R_{\delta}(e)$. 
Lemma 14. In a $\delta$-splitting, $S^{\prime}$, of a $\delta$-accurate approximate subdivision, $S$, for every endpoint $p \in S$, curve $e \in S$, and curve $e^{\prime} \in C(e), p \in L\left(e^{\prime}\right)$ implies $p<_{y} \overline{e_{+} \delta}$ and $p \in U\left(e^{\prime}\right)$ implies $p>_{y} \overline{e_{-\delta}}$.

Proof. We prove the $p \in L\left(e^{\prime}\right)$ case. Consider $e^{\prime}\left[a^{\prime}, b^{\prime}\right]$. If $p \in L(e), p<_{y} \overline{e_{+} \delta}$ by Lem. [1] If $p \in U(e), p$ is an endpoint of $f \in S$ with $e \prec_{y} f$. Thus, $p$ is an endpoint of a curve, $f^{\prime} \in C(f)$, but by Def. 9 $e^{\prime} \preceq_{y} f^{\prime}$, so $p \in U\left(e^{\prime}\right)$. By

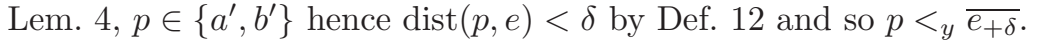

Definition $21\left(e_{-\delta}^{\prime}, e_{+\delta}^{\prime}, R_{\delta}^{\prime}\left(e^{\prime}\right)\right)$. For increasing $e^{\prime}\left[a^{\prime}, b^{\prime}\right] \in C(e)$, define $e_{-\delta}^{\prime}$ and $e_{+\delta}^{\prime}$ to be $e_{-\delta}$ and $e_{+\delta}$ restricted to $\left(a_{x}, b_{x}\right)$ if $e$ is increasing; otherwise $e_{-\delta}^{\prime}=e_{+\delta}^{\prime}=\emptyset$. Similarly for decreasing $e^{\prime}$. Define $R_{\delta}^{\prime}\left(e^{\prime}\right)=R\left(e^{\prime}\right)$ for horizontal or vertical $e^{\prime}$. For increasing $e^{\prime}, R_{\delta}^{\prime}\left(e^{\prime}\right)$ is $R\left(e^{\prime}\right)$ minus ${f^{\prime}}_{-\delta}^{-}$for increasing $f^{\prime} \preceq_{y} e^{\prime}$ and minus $g_{+\delta}^{+}$for increasing $g^{\prime} \succeq_{y} e^{\prime}$, and similarly for decreasing $e^{\prime}$.

Theorem 5. A consistent $\delta$-splitting of a $\delta$-accurate approximate subdivision is a $\delta$-refinement.

Proof. Modify the proof of Thm. पby replacing Lem.11with Lem. 14 to select a realization $r\left(e^{\prime}\right) \subset R_{\delta}^{\prime}\left(e^{\prime}\right)$ for each curve $e^{\prime}\left[a^{\prime}, b^{\prime}\right]$. If $e^{\prime}$ and $e$ are both increasing or both decreasing, $R_{\delta}^{\prime}\left(e^{\prime}\right)$ lies between $e_{-\delta}$ and $e_{+\delta}$ by construction hence $r\left(e^{\prime}\right)$ lies within $\delta$ of $e$. If $e$ is increasing but $e^{\prime}$ decreasing, we know $\operatorname{dist}\left(a^{\prime}, e\right)<\delta$ hence $a^{\prime}<_{y} e_{+\delta}$ hence $a^{\prime+-}<_{y} e_{+\delta}$. Similarly, $b^{\prime-+}>_{y} e_{-\delta}$. Hence, $r\left(e^{\prime}\right) \subset$ $a^{\prime+-} \cap b^{\prime-+}$ lies within $\delta$ of $e$.

Lemma 15. The refinement algorithm output is a $\delta$-splitting.

Proof. Let the algorithm split a curve, $e$, at an endpoint, $p$. We show that $\operatorname{dist}(p, e)<\delta$.

Step 1: Necessarily, $p \in L(e)$, because $p$ belongs to a group below $e$, and there is a violation of constraint 1 . Hence, there exists $q \in U(e)$ with $p_{x}=q_{x}$ and $p_{y} \geq q_{y}$. By Lem. 11] $p<_{y} e_{+\delta}$ and $q>_{y} e_{-\delta}$. Since $p_{y} \geq q_{y}, p>_{y} e_{-\delta}$ as well.

Step 2: For an incoming curve, $e[v, a]$, define $\mathrm{M}(e)$ analogously to $\mathrm{m}(e)$ as the maximum safe endpoint over $f[w, b] \preceq_{y} e$. Since $e \preceq_{y} e$ and $\operatorname{dist}(e, a) \leq$ $\operatorname{dist}(e, a), \mathrm{m}(e) \leq_{y} a \leq_{y} \mathrm{M}(e)$. Curve $e$ is split at the maximum $\mathrm{m}(f)$ with $f \preceq_{y} e$. Let $f[w, b]$ achieve this maximum. Since $e \preceq_{y} e, \mathrm{~m}(e) \leq_{y} \mathrm{~m}(f)$. If $b$ is safe for $e(\operatorname{dist}(b, e) \leq \operatorname{dist}(f, a)), \mathrm{m}(f) \leq_{y} b$ from above and $b \leq_{y} \mathrm{M}(e)$ by definition of $\mathrm{M}(e)$; otherwise, if $a$ is safe for $f, \mathrm{~m}(f) \leq_{y} a$ by definition of $\mathrm{m}(e)$ and $a \leq_{y} \mathrm{M}(e)$ from above. Hence $\mathrm{m}(f) \leq_{y} M(e)$. Since both $\mathrm{m}(e)$ and $\mathrm{M}(e)$ are safe for $e, \operatorname{dist}(\mathrm{m}(e), e), \operatorname{dist}(\mathrm{M}(e), e)<\delta$ by Lem. 16 below. The error bound neglects the distance computation error, $\gamma$, which is typically negligible with respect to $\delta$. Since we evaluate dist approximately, we might pick the endpoint that is unsafe by a little bit. Strictly speaking, we should replace $\delta$ with $\delta+2 \gamma$.

Step 3: We consider constraint 3 with $e$ increasing; the other cases are similar. Since $p \in L(e)$ and $d \in U(e), p<_{y} e_{+}$, and $d>_{y} e_{-\delta}$ by Lem.11 Also, $p>_{y} e_{-\delta}$ because $p_{x}<d_{x}, p_{y} \geq d_{y}$, and $e_{-\delta}$ is increasing by Lem. 9 . 
Step 4: $\operatorname{dist}(p, e)=0$.

Lemma 16. If $e[v, a] \prec_{y} f[w, b]$ violate constraint 2 at $a_{x}=b_{x}$, $\operatorname{dist}(a, f)<\delta$ or $\operatorname{dist}(b, e)<\delta$.

Proof. Let $\tilde{e}$ and $\tilde{f}$ be curves that show $\delta$-accuracy. Since $\tilde{e}<_{y} \tilde{f}$ and $a_{y}>b_{y}$, either $\tilde{e}$ has a vertical segment at $x=a_{x}$ that contains $b$ or $\tilde{f}$ has such a segment that contains $a$. The result follows because $\tilde{e}$ and $\tilde{f}$ are $\delta$-close to $e$ and $f$.

Theorem 6. The refinement algorithm computes a $\delta$-refinement.

Proof. Follows from Thm. 11 Thm. 5, and Lem.15.

\section{Set Operations}

We now turn to set operations. We compute the overlay approximate subdivision with our sweep algorithm[7] then refine. The sweep algorithm computes the curve crossing points approximately and preserves the curve endpoints, so the output endpoints are the union of the input endpoints and the approximate crossing points. The overlay $\prec y$ determines the membership of the overlay faces in the input faces via a standard algorithm. [15]

We modify the sweep algorithm to use red/blue sweep list insertion. When inserting a curve from the first input (red), find the two nearest red curves, using the red $\prec_{y}$, then compute the order of the new curve with respect to the intervening blue curves. Likewise for blue curve insertion. We avoid computation of red/red and blue/blue orders. We also ensure that the overlay $\prec_{y}$ agrees with the red and blue $\prec_{y}$, so overlay face membership is computed correctly.

\section{Euclidean Transformations}

We transform an approximate subdivision by transforming each curve, computing the transformed $\prec_{y}$, and invoking the refinement algorithm. The computational complexity is dominated by the refinement algorithm.

Translating by $t$ maps $e$ to $\{p+t \mid p \in e\}$ and drops each curve whose endpoints translate to the same point due to rounding. It does not change $\prec_{y}$ or $\delta$ accuracy. Scaling $(y)$ by $w$ maps $e$ to $\left\{\left(p_{x}, w p_{y}\right) \mid p \in e\right\}$ and drops the curves with equal endpoints. It does not change $\prec_{y}$, but scales $\delta$-accuracy by $w$. Skewing by $w$ maps $e$ to $\left\{\left(p_{x}, w p_{x}+p_{y}\right) \mid p \in e\right\}$ and splits the skewed curves at their $y$ turning points. It increases the distance between two points by at most a factor of $z=\sqrt{w^{2}+1}$. Skewing a deformation yields a deformation. Hence, skewing scales $\delta$-accuracy by at most $z$.

Rotation Rotating by $90^{\circ}$ maps $e$ to $\left\{\left(-p_{y}, p_{x}\right) \mid p \in e\right\}$. The rotated curves are monotone because the input curves are. The rotated $\prec_{y}$ is the horizontal order of the input curves. Let the rotations of $e$ and $f$ be $e^{r}$ and $f^{r}: e^{r} \prec_{y} f^{r}$ 


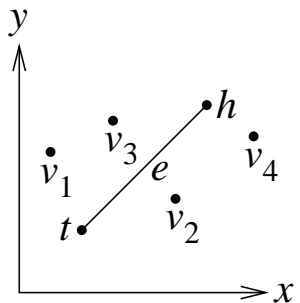

(a)

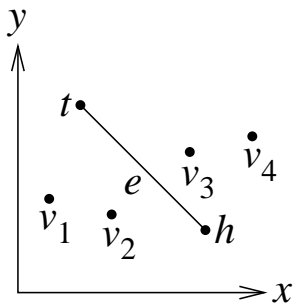

(b)

Figure 15: Horizontal endpoint $/ \prec_{y}$ for increasing (a) and decreasing (b) curves.

iff $e$ is left of $f$. It is $\delta$-accurate because a deformation that places two curves in $\prec_{y}$ order also places them in horizontal order.

Compute the horizontal order via a horizontal line sweep whose events are insertion and removal of each curve at its lower and upper endpoints. Removal is standard. A curve, $f$, is inserted by computing the order of its lower endpoint, $v$, with respect to $\mathrm{O}(\log n)$ curves in the sweep list. Let $e[t, h]$ be such a curve.

1. If $v_{x}<t_{x}, v$ is left of $e$.

2. If $f \prec_{y} e$ and $e$ is increasing, $v$ is right of $e$; if $e$ is decreasing, $v$ is left of $e$.

3. If $e \prec_{y} f$ and $e$ is increasing, $v$ is left of $e$; if $e$ is decreasing, $v$ is right of $e$.

4. If $h_{x}<v_{x}, v$ is right of $e$.

Fig. [15 shows points $v_{1}, \ldots, v_{4}$ that satisfy the four rules.

Rotating by $-90^{\circ}$ maps $e$ to $\left(e_{y},-e_{x}\right)$. The rotated $\prec_{y}$ is the opposite of the horizontal order of the input curves.

Rotation by $\theta$ is performed in two steps. Step 1 rotates the input by $90^{\circ}$ and replaces $\theta$ with $\theta-90^{\circ}$ for $45^{\circ}<\theta<135$, rotates the input by $-90^{\circ}$ and replaces $\theta$ with $\theta+90^{\circ}$ for $-135^{\circ}<\theta<-45^{\circ}$, and is omitted for other $\theta$ values. Let $s=\sin \theta$ and $c=\cos \theta$. Step 2 scales by $c$, skews by $s$, rotates by $90^{\circ}$, scales by $1 / c$, skews by $s / c$, and rotates by $-90^{\circ}$. The rotation formula is verified by direct calculation. The $\delta$-accuracy scales by

$$
1 \times c \times \sqrt{1+s^{2}} \times 1 \times \frac{1}{c} \times \sqrt{1+\frac{s^{2}}{c^{2}}} \times 1=\sqrt{\frac{1+s^{2}}{c^{2}}} .
$$

This factor is bounded by $\sqrt{3}$ because $|\theta| \leq 45^{\circ}$ after step 1 .

\section{Validation}

We have implemented set operations and Euclidean transformations for approximate subdivisions whose curves are approximate algebraic-curve segments. A 


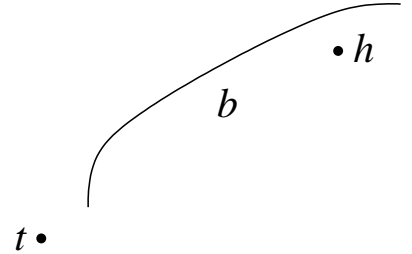

(a)

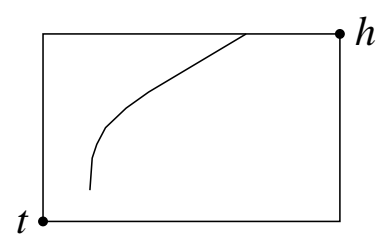

(b)

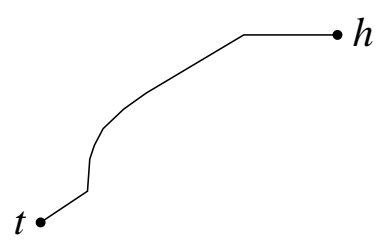

(c)

Figure 16: Curve representation (a), portion of $b$ in box (b), and curve (c).

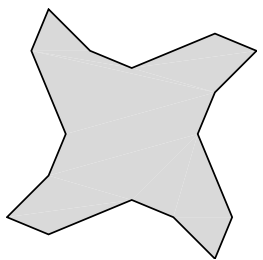

(a)

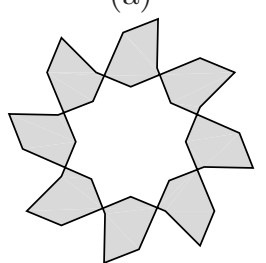

(e)

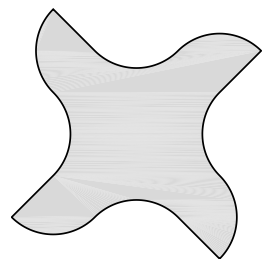

(b)

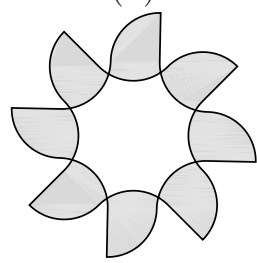

(f)

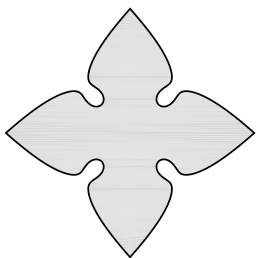

(c)

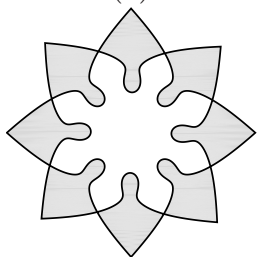

(g)

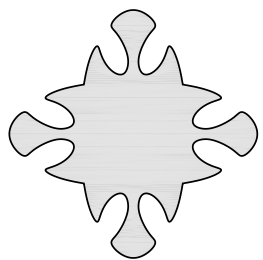

(d)

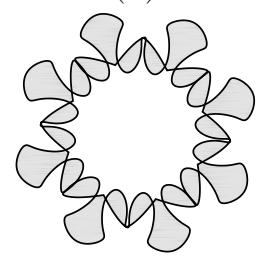

$\mathrm{h}$

Figure 17: Validation: shapes 1-4 (a-d) and their $s_{1}$ regions $(\mathrm{e}-\mathrm{h})$.

curve is represented by its endpoints, $t$ and $h$ with $t_{x} \leq h_{x}$, and by a monotone branch, $b$, of an algebraic curve. The curve is the portion of $b$ in the box with corners $t$ and $h$, plus two line segments that link $t$ and $h$ to it (Fig. 16). If $t_{y}<h_{y}$ and $b$ is decreasing or $h_{y}<t_{y}$ and $b$ is increasing, the curve is the line segment th. When a curve is split, the new curves inherit its branch. Our numerical solver [7] computes points on curves by solving univariate polynomials and computes curve crossing points by solving pairs of bivariate polynomials.

We validate the algorithms on sequences of set operations alternating with Euclidean transformations. We test the four shapes shown in Fig. 17, The shapes are drawn in the box $[-1,1] \times[-1,1]$. The curves are line segments in shape 1 , line and circle segments in shape 2 , degree- 3 curves in shape 3 , and degree- 6 curves in shape 4 . We initialize $s_{0}$ to a shape then set $s_{i+1}$ to the symmetric difference of $s_{i}$ with a Euclidean transform of $s_{i}$ for six iterations $(\mathrm{e}-\mathrm{h})$. The $s_{0}$ transform is translation by $(0.0123,0.0321)$ and rotation by 0.765 radians. These values are halved after each iteration.

The initial tests have high combinatorial complexity and no degeneracy. The program encounters no constraint violations, generates accurate outputs, and is 
Table 1: Initial tests: $n$ is the input size, $e$ is the output size, $\delta$ is the output error, $t$ is the running time in seconds, $c$ is the constraint enforcement percentage, and $s$ is the solver percentage.

\begin{tabular}{r|cccccc} 
shape & $n$ & $e$ & $\delta$ & $t$ & $c$ & $s$ \\
\hline 1 & 16 & 67000 & $3 \times 10^{-16}$ & 1.1 & 23 & 20 \\
2 & 20 & 70000 & $6 \times 10^{-16}$ & 3.6 & 10 & 70 \\
3 & 40 & 85000 & $8 \times 10^{-15}$ & 4.5 & 8 & 67 \\
4 & 80 & 277000 & $4 \times 10^{-12}$ & 127 & 1 & 91
\end{tabular}

fast. Table 1 summarizes the results. The input size, $n$, is the number of input curves. The output size, $e$, is the number of curves in the final, $s_{6}$, subdivision. The output error, $\delta$, is the larger of two error metrics both of which are the maximum over the six iterations. The first metric is the distance between the monotone branch of a curve and one of its endpoints. The second metric is the distance between a curve, $e$, and an endpoint, $v$, with $v \in L(e)$ and $v>_{y} e$, or $v \in U(e)$ and $v<_{y} e$. The running time, $t$, is for one core of an Intel Core 2 Duo. As the algebraic degree of the input increases from one to six, the constraint enforcement percentage, $c$, decreases rapidly and the numerical solver percentage, $s$, increases rapidly.

We repeated the tests with the $s_{0}$ Euclidean transformation divided by $2^{j}$ for $j=1, \ldots, 50$. As the transformation shrinks, the six iterates of the input shape converge, so the symmetric differences approach degeneracy. Fig. 18 plots the results for the initial, $j=0$, test and for the 50 subsequent tests. The output error and the solver percentage are omitted because they are essentially constant.

The output size decreases because each iterate has fewer intersections with the previous iterate. The number of constraint violations is zero for $j<15$, increases sharply from $j=25$ to $j=40$, then decreases to nearly zero. We expect that the curves become nearly identical rather than intersecting. The shape 3 running time increases from $j=24$, peaks at double the $j=0$ time at $j=32$, and decreases to nearly zero. The running time for the other shapes increases slightly if at all then decreases to nearly zero. The running time for shape 4 is divided by ten, so it can be displayed with the others. The percentage spent on constraint enforcement decreases, except for a peak in $40<j<50$ where the output is small relative to the number of violations. The percentage also decreases as the input degree increases: constraint enforcement, which is combinatorial, is independent of degree, whereas curve intersection, which is numerical, is polynomial in degree.

\section{Conclusions}

This paper presents approximate planar shape-manipulation algorithms. The validation results show that the algorithms are accurate and fast on both generic and degenerate inputs. Constraint enforcement takes a small fraction of the 

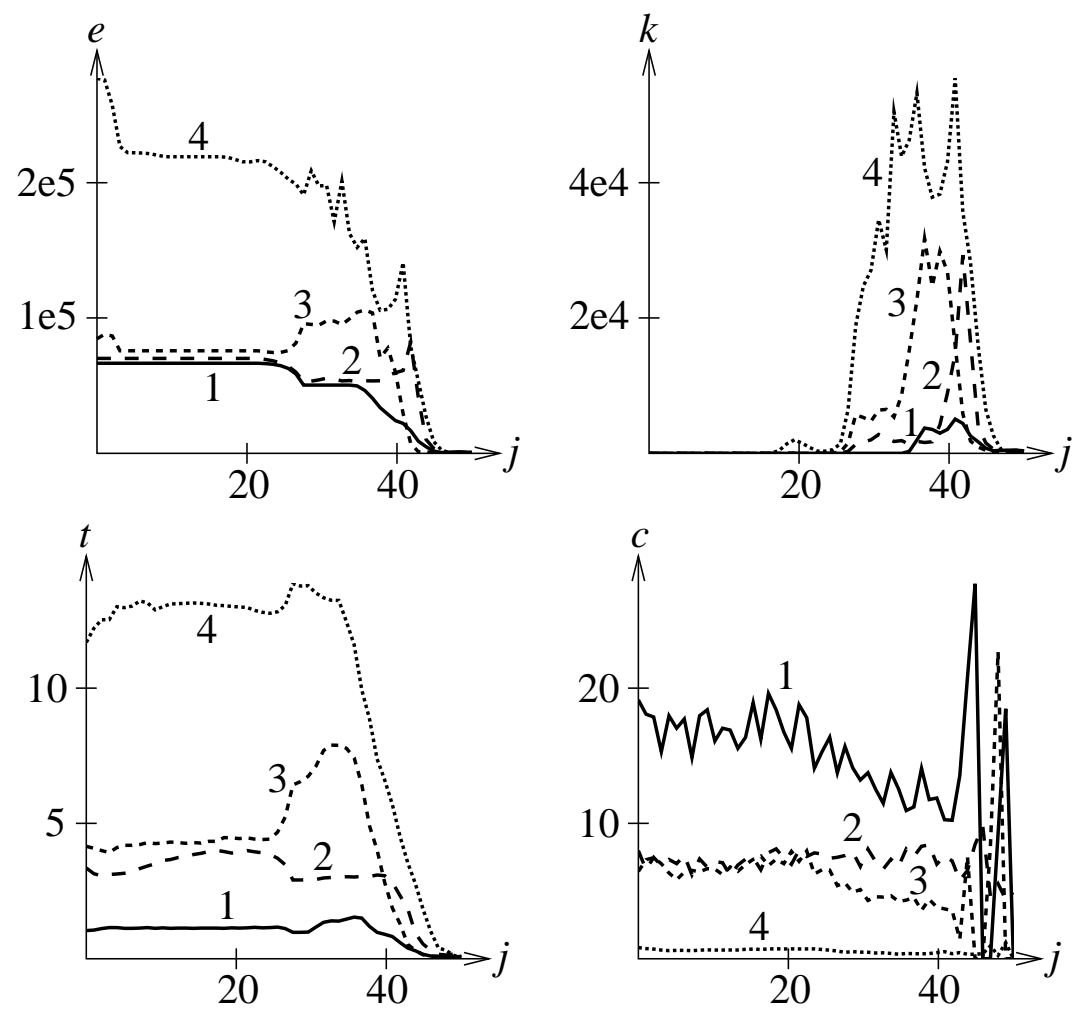

Figure 18: All tests: $j$ is the iteration, $e$ is the output size, $k$ is the number of constraint violations, $t$ is the running time, and $c$ is the constraint enforcement percentage. 
running time, whereas numerical computation takes most of the time. The results support our thesis that inconsistency sensitive algorithms are efficient and accurate.

\section{Acknowledgments}

Research supported by NSF grants IIS-0082339, CCF-0306214, and CCF-0304955.

\section{References}

[1] Chee Yap. Robust geometric computation. In J. E. Goodman and J. O'Rourke, editors, Handbook of discrete and computational geometry, chapter 41, pages 927-952. CRC Press, Boca Raton, FL, second edition, 2004.

[2] Computational geometry algorithms library. http://www.cgal.org/.

[3] Victor Milenkovic. Shortest path geometric rounding. Algorithmica, 27(1):57-86, 2000.

[4] Dan Halperin and Eli Packer. Iterated snap rounding. Computational Geometry: Theory and Applications, 23(2):209-222, 2002.

[5] Arno Eigenwillig, Lutz Kettner, and Nicola Wolpert. Snap rounding of bézier curves. In SCG '0\%: Proceedings of the twenty-third annual symposium on Computational geometry, pages 158-167, New York, NY, USA, 2007. ACM.

[6] S. Fortune. Polyhedral modelling with multiprecision integer arithmetic. Computer-Aided Design, 29(2):123-133, 1997.

[7] Victor Milenkovic and Elisha Sacks. An approximate arrangement algorithm for semi-algebraic curves. International Journal of Computational Geometry and Applications, 17(2), 2007.

[8] Victor Milenkovic and Elisha Sacks. Two approximate minkowski sum algorithms. International Journal of Computational Geometry and Applications, 20(4):485-509, 2010.

[9] Sigal Raab. Controlled perturbation for arrangements of polyhedral surfaces. In Proceedings of the 15th Symposium on Computational Geometry, pages 163-172. ACM, 1999.

[10] Dan Halperin and Christian Shelton. A perturbation scheme for spherical arrangements with application to molecular modeling. Computational Geometry: Theory and Applications, 10(4):273-288, 1998. 
[11] Dan Halperin and Eran Leiserowitz. Controlled perturbation for arrangements of circles. International Journal of Computational Geometry and Applications, 14(4-5):277-310, 2004.

[12] Stefan Funke, Christian Klein, Kurt Mehlhorn, and Susanne Schmitt. Controlled perturbation for delaunay triangulations. In SODA '05: Proceedings of the sixteenth annual ACM-SIAM symposium on Discrete algorithms, pages 1047-1056, Philadelphia, PA, USA, 2005. ACM, Society for Industrial and Applied Mathematics.

[13] Elisha Sacks, Victor Milenkovic, and Min-Ho Kyung. Controlled linear perturbation. Computer-Aided Design, 43(10):1250-1257, 2011.

[14] Victor Milenkovic and Elisha Sacks. Robust complete path planning in the plane. In Proceedings of the Workshop on the Algorithmic Foundations of Robotics. WAFR, 2012.

[15] Mark de Berg, Otfried Cheong, Marc van Kreveld, and Mark Overmars. Computational Geometry: Algorithms and Applications. Springer, Berlin, third edition, 2008. 